Dhaka Univ. J. Sci. 64(2): 91-98, 2016 (July)

\title{
Unsteady MHD Free Convection and Mass Transfer Flow Through a Vertical Oscillatory Porous Plate in a Rotating Porous Medium with Hall, Ion-slip Currents and Heat Source
}

\author{
Md. Delowar Hossain ${ }^{1}$, Md. Abdus Samad ${ }^{1}$ and Md. Mahmud Alam² \\ ${ }^{I}$ Deparment of Applied Mathematics, Dhaka University, Dhaka-1000, Bangladesh \\ ${ }^{2}$ Mathematics Discipline, Khulna University, Khulna-9208, Bangladesh
}

(Received: 3 March 2015; Accepted: 21 March 2016)

\begin{abstract}
The analytical solution is made on the unsteady flow of an electrically conducting viscous incompressible fluid bounded by an infinite vertical porous plate. The plate executes harmonic oscillation at a frequency $n$ in its own plane. The governing equations of the problem contain coupled partial differential equations. The dimensionless equations are solved analytically using perturbation technique. The effect of various parameters of the problem on the velocity, temperature and concentration field within the boundary layer are discussed and shown graphically.
\end{abstract}

Keywords: MHD flow, Hall current, Ion-slip current, Suction parameter, Heat source parameter.

\section{Introduction}

The study of MHD flow with Hall currents is important for many engineering applications. On the other hand, present interest in the study of magneto hydrodynamic fluid flow in a rotating system has been motivated by several important problems, such as maintenance and secular variations of earth's magnetic field, the internal rotation rate of sun, the structure of rotating stars, the planetary and solar dynamo problem, centrifugal machines etc. Hence it is now proposed to study the MHD free convection flow of a partiallyionized gas past an infinite vertical porous plate in a rotating system when a strong magnetic field of a uniform strength is applied perpendicularly to the flow. The generalized Ohm's law with Hall and ion-slip currents has been used. The study of oscillatory flow, which has many practical applications, was first initiated by Lighthill ${ }^{1}$. The oscillatory flow of a viscous incompressible fluid past an infinite vertical plate was studied by Soundalgekar ${ }^{2}$. Soundalgekar ${ }^{3}$ also studied the problem of two dimensional unsteady flow of an electrically conducting fluid past an infinite vertical porous plate with uniform suction at the plate. Mittal and Bhat ${ }^{4}$ have discussed the heat transfer by developing MHD flow with Hall and ion-slip currents. Soundalgekar et al. ${ }^{5}$ have presented the Hall and ion-slip effects in MHD coutte flow with heat transfer. The effects of hall currents on the oscillatory MHD flow past a flat plate are investigated by Venkatasiva Murthy ${ }^{6}$. Singh ${ }^{7}$ studied the effects of Hall currents on an oscillatory MHD flow in Stokes problem past an infinite vertical porous plate. $\operatorname{Ram}^{8}$ studied wall temperature oscillation on convection flow in a rotating system with hall and ion-slip currents. Combined effects of hall and ion-slip currents on free convective heat generating flow past a semi-infinite vertical flat plate have been investigated by Abo-Eldahab et al. ${ }^{9}$. Attia ${ }^{10}$ has discussed the combined effects of hall and ion-slip current of a conducting fluid flow due to a rotating disk. Maji et al. ${ }^{11}$ studies Hall effects on hydromagnetic flow on an oscillating porous plate.

Hence our aim is to study the effects of hall and ion-slip currents on the unsteady MHD flow due to oscillations of an infinite non-conducting vertical porous plate in a viscous incompressible electrically conducting fluid. Also the effects of Hall currents, the free convection and the oscillatory free stream on the flow field are studied.

\section{Governing Equations}

The unsteady flow of an electrically conducting incompressible viscous fluid past an infinite vertical porous plate $y=0$, with the $x$-axis chosen along the plate, when the plate velocity $U(t)$ oscillates in time $t$ with a frequency $n$ and is given as $U(t)=U_{0}(1+\cos n t)$ is considered. The flow is assumed to be in the $x$-direction, is taken along the plate in the upward direction normal to the $y$-axis. Initially the fluids as well as the plate are at rest but for time $t>0$ the whole system is allowed to rotate with a constant angular velocity $\Omega$ about the $y$-axis. The plate temperature and concentration are instantly rise to $T_{w}\left(>T_{\infty}\right)$ and $C_{w}\left(>C_{\infty}\right)$, which are thereafter maintained constant, where $T_{\infty}$ and $C_{\infty}$ are the temperature and concentration of the uniform flow. The physical configuration of the problem is shown in Fig. 1.

A transverse strong magnetic field $B_{0}$ with constant intensity is imposed along the $y$ axis. For electrically conducting fluid, Hall and ion-slip currents will significantly affect the flow in presence of large magnetic field. The effect of Hall current gives rise to force in the $z$ direction which induces a cross flow in that direction

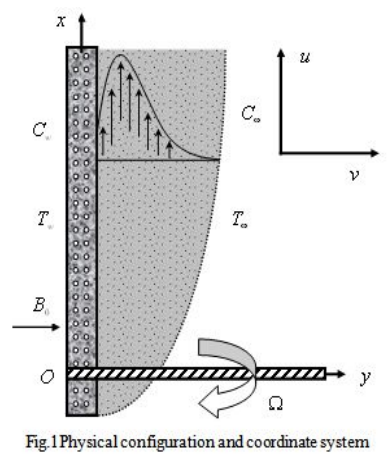
and hence the flow becomes three dimensions. If $\mathbf{J}=\left(J_{x}, J_{y}, J_{z}\right)$ is the current density, from the relation $\bar{\nabla} . \mathbf{J}=0, J_{y}=$ constant has been obtained. Since the

* Author for correspondence. e-mail: alam_mahmud2000@yahoo.com 
plate is electrically non-conducting, $J_{y}=0$ at the plate and hence zero everywhere. Since the plate is infinite in extent, all physical quantities, except pressure, are functions of $y$ and $t$ only. The governing boundary layer equations may be written as follows:

Continuity equation: $\frac{\partial v}{\partial y}=0$

Momentum equations:

$$
\begin{gathered}
\frac{\partial u}{\partial t}+v \frac{\partial u}{\partial y}=v \frac{\partial^{2} u}{\partial y^{2}}+g \beta\left(T_{w}-T_{\infty}\right)+g \beta^{*}\left(C_{w}-C_{\infty}\right) \\
+2 \Omega w-\frac{v}{k} u-\frac{\sigma_{e} B_{0}^{2}}{\rho\left(\alpha_{e}^{2}+\beta_{e}^{2}\right)}\left(\alpha_{e} u+\beta_{e} w\right) \\
\frac{\partial w}{\partial t}+v \frac{\partial w}{\partial y}=v \frac{\partial^{2} w}{\partial y^{2}}-2 \Omega u-\frac{v}{k} w \\
+\frac{\sigma_{e} B_{0}^{2}}{\rho\left(\alpha_{e}^{2}+\beta_{e}^{2}\right)}\left(\beta_{e} u-\alpha_{e} w\right)
\end{gathered}
$$

Energy equation:

$$
\frac{\partial T}{\partial t}+v \frac{\partial T}{\partial y}=\frac{\sigma}{\rho c_{p}} \frac{\partial^{2} T}{\partial y^{2}}+Q^{*}\left(T_{w}-T\right)
$$

Mass equation:

$$
\frac{\partial C}{\partial t}+v \frac{\partial C}{\partial y}=D_{m} \frac{\partial^{2} C}{\partial y^{2}}+\frac{D_{m} k_{T}}{T_{m}} \frac{\partial^{2} T}{\partial y^{2}}
$$

The initial and boundary conditions for the model are as follows:

$$
\begin{aligned}
& u(y, t)=0, w(y, t), T(y, t)=T_{\infty} \\
& C(y, t)=C_{\infty} \text { for } t \leq 0 \\
& u(y, t)=U_{0}\left[1+\frac{\varepsilon}{2}\left(e^{\text {int }}+e^{- \text {int }}\right)\right], w(y, t)=0, \\
& T(y, t)=T_{w}, C(y, t)=C_{w} \text { at } y=0, t>0 \\
& u(y, t)=0, w(y, t)=0, T(y, t)=T_{\infty}, C(y, t)=C_{\infty} \\
& \text { at } y \rightarrow \infty, t>0
\end{aligned}
$$

where $\varepsilon<<1, U_{0}$ is uniform velocity, $\alpha=1+\beta_{e} \beta_{i}$, $\beta_{e}$ is Hall current parameter, $\beta_{i}$ is ion-slip current parameter, $y$ is Cartesian co-ordinate, $u$ and $w$ are the components of flow velocity, $g$ is the local acceleration due to gravity, $\beta$ is the thermal expansion coefficient, $\beta^{*}$ is the concentration expansion coefficient, $v$ is the kinematic viscosity, $k$ is the magnetic permeability, $\rho$ is the density of the fluid, $\sigma_{e}$ is the electrical conductivity, $\sigma$ is the thermal conductivity, $c_{p}$ is the specific heat at the constant pressure, $D_{m}$ is the coefficient of mass diffusivity, $Q^{*}$ is the heat absorption quantity, $k_{T}$ is the thermal diffusion ratio, $T_{m}$ is the mean fluid temperature and $B_{0}$ is the magnetic component in $y$-direction. Now a convenient solution of equation (1) is

$v=-v_{0}$ (constant)

where the constant velocity $v_{0}$ acting normal to the plate which is positive for suction and negative for blowing. Using equation (8), the equations (2)-(5) become:

$$
\begin{aligned}
& \frac{\partial u}{\partial t}-v_{0} \frac{\partial u}{\partial y}=v \frac{\partial^{2} u}{\partial y^{2}}+g \beta\left(T_{w}-T_{\infty}\right)+g \beta^{*}\left(C_{w}-C_{\infty}\right) \\
& +2 \Omega w-\frac{v}{k} u-\frac{\sigma_{e} B_{0}^{2}}{\rho\left(\alpha_{e}^{2}+\beta_{e}^{2}\right)}\left(\alpha_{e} u+\beta_{e} w\right)
\end{aligned}
$$

$$
\begin{aligned}
\frac{\partial w}{\partial t}-v_{0} \frac{\partial w}{\partial y} & =v \frac{\partial^{2} w}{\partial y^{2}}-2 \Omega u-\frac{v}{k} w \\
+ & \frac{\sigma_{e} B_{0}^{2}}{\rho\left(\alpha_{e}^{2}+\beta_{e}^{2}\right)}\left(\beta_{e} u-\alpha_{e} w\right)
\end{aligned}
$$

$\frac{\partial T}{\partial t}-v_{0} \frac{\partial T}{\partial y}=\frac{\sigma}{\rho c_{p}} \frac{\partial^{2} T}{\partial y^{2}}+Q^{*}\left(T_{w}-T\right)$

$\frac{\partial C}{\partial t}-v_{0} \frac{\partial C}{\partial y}=D_{m} \frac{\partial^{2} C}{\partial y^{2}}+\frac{D_{m} k_{T}}{T_{m}} \frac{\partial^{2} T}{\partial y^{2}}$

The initial and boundary conditions for the model are as follows:

$t<<0, u(y, t)=0, w(y, t)=0, T(y, t)=T_{\infty}$,

$C(y, t)=C_{\infty}$ everywhere

$$
\begin{aligned}
& u(y, t)=U_{0}\left[1+\frac{\varepsilon}{2}\left(e^{\mathrm{int}}+e^{-\mathrm{int}}\right)\right], w(y, t)=0, \\
& T(y, t)=T_{w}, C(y, t)=C_{w} \text { at } y=0, t>0 \\
& u(y, t)=0, w(y, t)=0, T(y, t)=T_{\infty}, \\
& C(y, t)=C_{\infty} \quad \text { at } y \rightarrow \infty, t>0
\end{aligned}
$$

\section{Mathematical Formulations}

For the purpose of solving the system of equation analytically, transformation of the governing equations are needed to obtain the non-dimension form, the usual nondimensional variables are introduced as follows:

$$
\begin{aligned}
& Y=\frac{y U_{0}}{v}, f=\frac{u}{U_{0}}, g=\frac{w}{U_{0}}, \tau=\frac{t U_{0}^{2}}{v}, \omega=\frac{n v}{U_{0}^{2}}, \\
& \theta=\frac{T-T_{\infty}}{T_{w}-T_{\infty}}, \phi=\frac{C-C_{\infty}}{C_{w}-C_{\infty}}
\end{aligned}
$$


The non-dimensional system of coupled equations have been obtained by using the above mentioned nondimensional variables in equations (9)-(12),

$$
\begin{aligned}
& \frac{\partial f}{\partial \tau}-\lambda \frac{\partial f}{\partial Y}=\frac{\partial^{2} f}{\partial Y^{2}}+G_{r} \theta+G_{m} \phi+2 R g-\gamma f \\
& -\frac{M\left(\alpha_{e} f+\beta_{e} g\right)}{\left(\alpha_{e}^{2}+\beta_{e}^{2}\right)} \\
& \frac{\partial g}{\partial \tau}-\lambda \frac{\partial g}{\partial Y}=\frac{\partial^{2} g}{\partial Y^{2}}-2 R f-\gamma g+\frac{M\left(\beta_{e} f-\alpha_{e} g\right)}{\left(\alpha_{e}^{2}+\beta_{e}^{2}\right)} \\
& \frac{\partial \theta}{\partial \tau}-\lambda \frac{\partial \theta}{\partial Y}=\frac{1}{P_{r}} \frac{\partial^{2} \theta}{\partial Y^{2}}-\frac{\alpha}{P_{r}} \theta \\
& \frac{\partial \phi}{\partial \tau}-\lambda \frac{\partial \phi}{\partial Y}=\frac{1}{S_{c}} \frac{\partial^{2} \phi}{\partial Y^{2}}+S_{0} \frac{\partial^{2} \theta}{\partial Y^{2}}
\end{aligned}
$$

The corresponding boundary conditions are as follows:

$f=0, g=0, \theta=0, \phi=0$ for $\tau \leq 0$

$f=1+\frac{\varepsilon}{2}\left(e^{\mathrm{i} \omega \tau}+e^{-\mathrm{i} \omega \tau}\right), g=0, \theta=1, \phi=1$ at $\left.Y=0\right\}$

$f=0, g=0, \theta=0, \phi=0 \quad$ at $Y \rightarrow \infty, \tau>0\}$

where $\lambda=\frac{v_{0}}{U_{0}} \quad$ (Suction Parameter),

$G_{r}=\frac{g \beta\left(T_{w}-T_{\infty}\right) v}{U_{0}^{3}}$ (Grashof Number),

$G_{m}=\frac{g \beta^{*}\left(C_{w}-C_{\infty}\right) v}{U_{0}^{3}} \quad$ (Modified Grashof Number),

$M=\frac{\sigma B_{0}^{2} v}{\rho U_{0}^{2}}$ (Magnetic Parameter), $P_{r}=\frac{\rho c_{p} v}{\sigma}$ (Prandtl

Number), $S_{c}=\frac{v}{D_{m}}$ (Schmidt Number),

$S_{0}=\frac{D_{m} k_{T}\left(T_{w}-T_{\infty}\right)}{v T_{m}\left(C_{w}-C_{\infty}\right)}$ (Soret Number), $R=\frac{\Omega v}{U_{0}^{2}}$

(Rotational Parameter), $\alpha=\frac{Q v^{2} \rho c_{p}}{U_{0}^{2} \sigma}$ (Heat Source

Parameter), $\gamma=\frac{v^{2}}{k U_{0}^{2}}$ (Permeably of the Porous

Medium),

The equations (15)-(16) have been further simplified by putting the fluid velocity in the complex form $Q(Y, \tau)=(f+i g)$ and the following equations have been obtained as follows: $\frac{\partial Q}{\partial \tau}-\lambda \frac{\partial Q}{\partial Y}=\frac{\partial^{2} Q}{\partial Y^{2}}+G_{r} \theta+G_{m} \phi-\xi Q$

where $Q=(f+i g), \xi=\gamma+2 i R+\frac{M\left(\alpha_{e}-\beta_{e}\right)}{\alpha_{e}^{2}+\beta_{e}^{2}}$

The corresponding boundary conditions (19 and (20) are now transformed in the following form:

$$
\begin{aligned}
& \frac{\partial Q}{\partial \tau}-\lambda \frac{\partial Q}{\partial Y}=\frac{\partial^{2} Q}{\partial Y^{2}}+G_{r} \theta+G_{m} \phi-\xi Q \\
& \frac{\partial \theta}{\partial \tau}-\lambda \frac{\partial \theta}{\partial Y}=\frac{1}{P_{r}} \frac{\partial^{2} \theta}{\partial Y^{2}}-\frac{\alpha}{P_{r}} \theta \\
& \frac{\partial \phi}{\partial \tau}-\lambda \frac{\partial \phi}{\partial Y}=\frac{1}{S_{c}} \frac{\partial^{2} \phi}{\partial Y^{2}}+S_{0} \frac{\partial^{2} \theta}{\partial Y^{2}}
\end{aligned}
$$

The corresponding boundary conditions (19) and (20) are now transformed in the following from:

$Q=0, \theta=0, \phi=0$ for $\tau \leq 0$

$$
\left.\begin{array}{l}
Q=1+\frac{\varepsilon}{2}\left(e^{i \omega \tau}+e^{-i \omega \tau}\right), \theta=1, \phi=1 \quad \text { at } Y=0 \\
Q=0, \quad \theta=0, \phi=0 \quad \text { at } Y \rightarrow \infty, \tau>0
\end{array}\right\}
$$

\section{Solution Technique}

In order to solve the equation (21)-(23) with the boundary condition (25) in the neighborhood of the plate under the above boundary conditions (24) and (25), the perturbation technique has been considered as follows:

$$
\begin{aligned}
& Q(Y, \tau)=Q_{0}(Y)+\frac{\varepsilon}{2}\left[e^{i \omega \tau} Q_{1}(Y)+e^{-i \omega \tau} Q_{2}(Y)\right] \\
& \theta(Y, \tau)=\theta_{0}(Y)+\frac{\varepsilon}{2}\left[e^{i \omega \tau} \theta_{1}(Y)+e^{-i \omega \tau} \theta_{2}(Y)\right] \\
& \phi(Y, \tau)=\phi_{0}(Y)+\frac{\varepsilon}{2}\left[e^{i \omega \tau} \phi_{1}(Y)+e^{-i \omega \tau} \phi_{2}(Y)\right]
\end{aligned}
$$

Using equations (26)-(28) into equations (21)-(23), equating the harmonic and non-harmonic terms and neglecting higher order terms, the obtained equations are as follows:

$\frac{\partial^{2} Q_{0}}{\partial Y^{2}}+\lambda \frac{\partial Q_{0}}{\partial Y}-\xi Q_{0}+G_{r} \theta_{0}+G_{m} \phi_{0}=0$

$\frac{\partial^{2} Q_{1}}{\partial Y^{2}}+\lambda \frac{\partial Q_{1}}{\partial Y}+G_{r} \theta_{1}+G_{m} \phi_{1}-(\xi+i \omega) Q_{1}=0$

$\frac{\partial^{2} Q_{2}}{\partial Y^{2}}+\lambda \frac{\partial Q_{2}}{\partial Y}+G_{r} \theta_{2}+G_{m} \phi_{2}-(\xi-i \omega) Q_{2}=0$

$\frac{\partial^{2} \theta_{0}}{\partial Y^{2}}+\lambda P_{r} \frac{\partial \theta_{0}}{\partial Y}-\alpha \theta_{0}=0$ 


$$
\begin{aligned}
& \frac{\partial^{2} \theta_{1}}{\partial Y^{2}}+\lambda P_{r} \frac{\partial \theta_{1}}{\partial Y}-\left(\alpha+i \omega P_{r}\right) \theta_{1}=0 \\
& \frac{\partial^{2} \theta_{2}}{\partial Y^{2}}+\lambda P_{r} \frac{\partial \theta_{2}}{\partial Y}-\left(\alpha-i \omega P_{r}\right) \theta_{2}=0 \\
& \frac{\partial^{2} \phi_{0}}{\partial Y^{2}}+\lambda S_{c} \frac{\partial \phi_{0}}{\partial Y}+S_{0} S_{c} \frac{\partial^{2} \theta_{0}}{\partial Y^{2}}=0 \\
& \frac{\partial^{2} \phi_{1}}{\partial Y^{2}}+\lambda S_{c} \frac{\partial \phi_{1}}{\partial Y}-i \omega S_{c} \phi_{1}+S_{0} S_{c} \frac{\partial^{2} \theta_{1}}{\partial Y^{2}}=0 \\
& \frac{\partial^{2} \phi_{2}}{\partial Y^{2}}+\lambda S_{c} \frac{\partial \phi_{2}}{\partial Y}+i \omega S_{c} \phi_{2}+S_{0} S_{c} \frac{\partial^{2} \theta_{2}}{\partial Y^{2}}=0
\end{aligned}
$$

The appropriate boundary conditions for the equations (24)(25) are as follows:

$$
\begin{aligned}
& Q_{0}=Q_{1}=Q_{2}=1, \theta_{0}=1, \theta_{1}=\theta_{2}=0, \phi_{0}=1 \\
& \phi_{1}=\phi_{2}=0 \text { at } Y=0 \\
& Q_{0}=Q_{1}=Q_{2}=0, \theta_{0}=\theta_{1}=\theta_{2}=0, \\
& \phi_{0}=\phi_{1}=\phi_{2}=0 \quad \text { at } Y \rightarrow \infty
\end{aligned}
$$

Solving equations (29)-(37) by using boundary conditions (38)and (39), the following equations have been obtained:

$$
\begin{aligned}
Q= & B_{5} e^{-A_{7} Y}-B_{3} e^{-A_{2} Y}-B_{4} e^{-A_{3} Y} \\
& +\frac{\varepsilon}{2} e^{-i \omega \tau} e^{-A_{13} Y}+\frac{\varepsilon}{2} e^{-i \omega \tau} e^{-A_{19} Y} \\
\theta= & e^{-A_{2} Y} \\
\phi= & A_{4} e^{-A_{2} Y}+A_{5} e^{-A_{3} Y}
\end{aligned}
$$

where

$$
\begin{aligned}
& A_{2}=\frac{\lambda P_{r}+\sqrt{\left(\lambda P_{r}\right)^{2}+4 \alpha}}{2}, A_{3}=\lambda S_{c}, A_{4}=\frac{S_{0} S_{c} A_{2}}{\lambda S_{c}-A_{2}}, \\
& A_{5}=\left(1-A_{4}\right), A_{7}=\frac{\lambda+\sqrt{\lambda^{2}+4 \xi}}{2}, B_{2}=A_{5} G_{m}, \\
& A_{13}=\frac{\lambda+\sqrt{\lambda^{2}+4 B_{8}}}{2}, \xi=\gamma+2 i R+\frac{M\left(\alpha_{e}-\beta_{e}\right)}{\alpha_{e}^{2}+\beta_{e}^{2}}, \\
& A_{19}=\frac{\lambda+\sqrt{\lambda^{2}+4 B_{10}}}{2}, B_{1}=G_{r}+A_{4} G_{m}, \\
& B_{3}=\frac{B_{1}}{A_{2}^{2}-\lambda A_{2}-\xi}, B_{4}=\frac{B_{2}}{A_{3}^{2}-\lambda A_{3}-\xi}, \\
& B_{5}=1+B_{3}+B_{4}, B_{8}=\xi+i \omega, B_{10}=\xi-i \omega
\end{aligned}
$$

\section{Results and Discussion}

The non-dimensional velocity components $f$ and $g$ are commonly known as the primary and secondary velocities. Numerical results are presented in Figs.2-19. These results show the effect of the material parameters on the quantities mentioned in section III. The most important fluids are atmospheric air, salt water and fresh water and the results are limited to $P_{r}=0.71$ (Prandtl number for air at $20^{\circ} \mathrm{C}$ ), to $P_{r}=1.00$ (Prandtl number for salt water at $20^{\circ} \mathrm{C}$ ), $P_{r}=7.00$ (Prandtl number for water at $20^{\circ} \mathrm{C}$ ). For the purpose of computation, $\omega \tau=\frac{\pi}{2}, \varepsilon=0.01$ have been chosen arbitrarily. Minor effects on velocity, temperature and concentration distributions are not shown for brevity.

It is observed that in Fig.2, primary velocity increases with the increase of Hall parameter $\beta_{e}$. This is because the effective conductivity decreases with increasing $\beta_{e}$ which reduces the magnetic damping force on primary velocity. In Fig.3, the secondary velocity profiles are shown and it is seen from this figure there is an opposite effect to increase the Hall parameter. From Fig.4, it is seen that secondary velocity decreases with the increase of ion-slip parameter $\beta_{i}$

Figs.5-7, represent the effect of Prandtl number $P_{r}$ on primary velocity, secondary velocity, temperature.

In Fig.5, primary velocity profiles decrease with an increase of $P_{r}$. This is because of the free convection the surface velocity is higher than the adjacent fluid velocity and the momentum boundary thickness decreases. But opposite behavior is seen for secondary velocity in Fig.6. From Fig.7, it has been seen that temperature profiles decrease with the increases of $P_{r}$. If $P_{r}$ increases, the thermal diffusivity decreases and these phenomena lead to the decreasing of energy ability that reduces the thermal boundary layer.

From Fig. 8 , it is seen that the primary velocity decreases with an increase of permeability parameter $\gamma$. This is due to the fact that increasing the value of $\gamma$ has tendency to resist the flow causing to reduce the thickness of the boundary layer. But the secondary velocity has reverse effects due to increase of $\gamma$ which is found in Fig. 9.

It is clearly seen that from in Fig.10, the primary velocity decreases due to increase of magnetic parameter $M$. The presence of magnetic field in an electrically conducting fluid introduces a force called Lorentz force. This force has tendency to slow down the motion of the fluid. Whereas the secondary velocity increases with an increase of $M$ which is shown in Fig.11.

From Fig.12, the primary velocity is decreased with an increase of rotational parameter $R$. Rotation parameter defines the relative magnitude of the Coriolis force and the viscous force, thus rotation retards primary flow. Similar behavior is found for secondary velocity in Fig. 13.

From Fig.14, it is seen that the primary velocity decreases with increase in the heat source parameter $\alpha$ because when heat is absorbed the buoyancy force decreases which retards 
the flow. But the secondary velocity has opposite behavior is seen in Fig.15. It is clearly seen that in Fig.16, the temperature distribution decreases due to increase of $\alpha$. Because when heat is absorbed, the buoyancy force decreases the temperature profiles.

Fig.17 and Fig.19 depict the primary velocity and concentration distributions for different values of the Soret

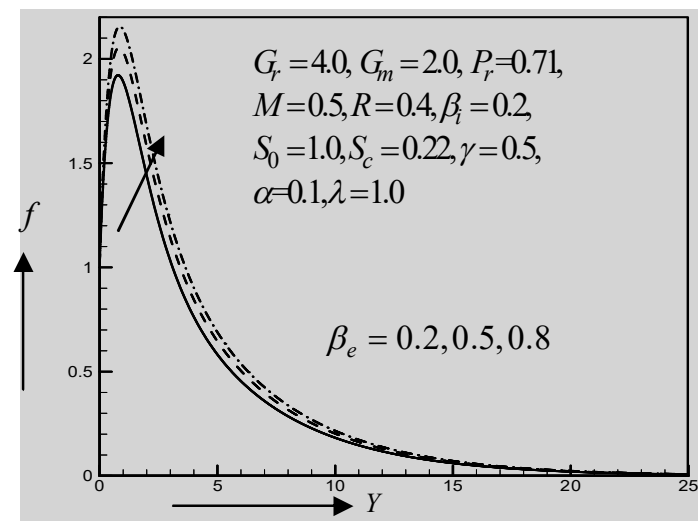

Fig. 2. Primary velocity profile for different values of Hall parameter $\beta_{e}$

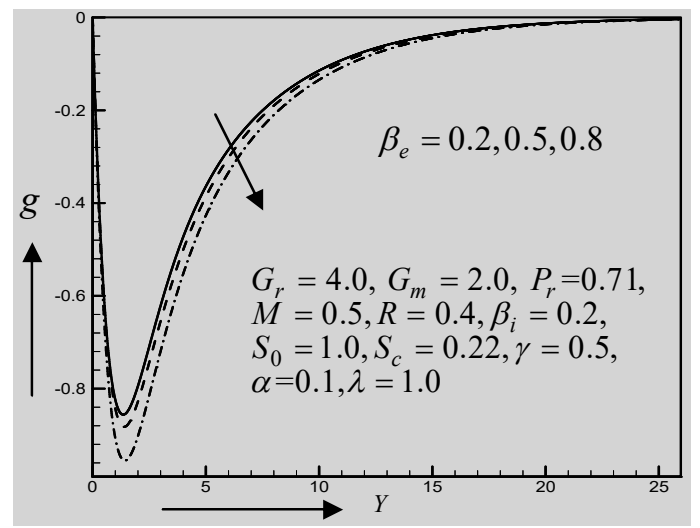

Fig. 3. Secondary velocity profile for different values of Hall parameter $\beta_{e}$

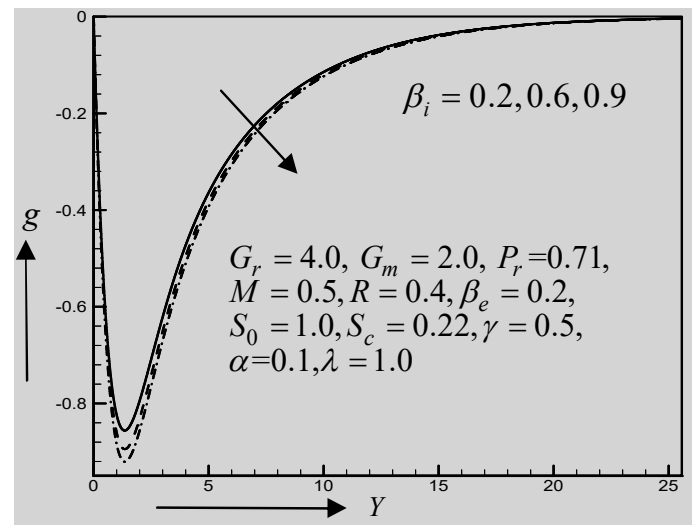

Fig. 4. Secondary velocity profile for different values of ion-slip parameter $\beta_{i}$ number $S_{0}$. The Soret number $S_{0}$ defines the effect of the temperature gradients inducing significant mass diffusion effects. It is noticed that an increase in the $S_{0}$ results in an increase in the primary velocity and concentration within the boundary layer. Whereas secondary velocity decreases with an increase of $S_{0}$ which is seen in Fig. 18.

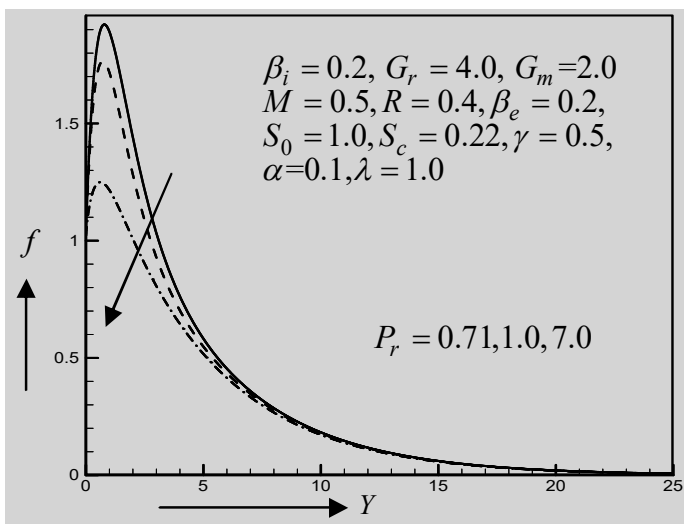

Fig. 5. Primary velocity profile for different values of Prandtl number $P_{r}$

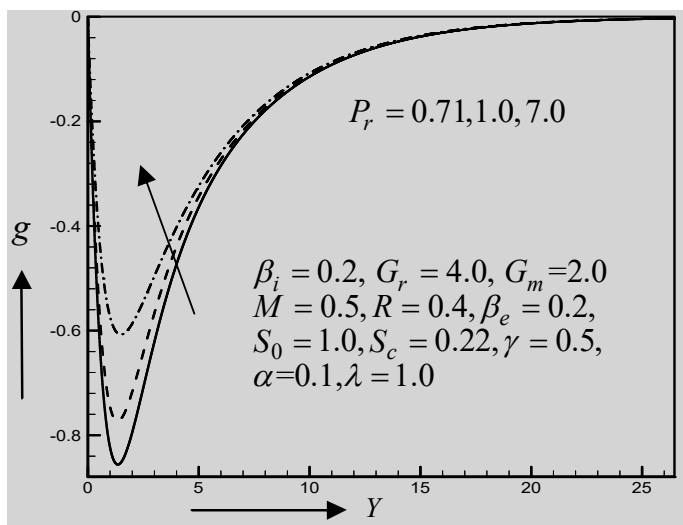

Fig. 6. Secondary velocity profile for different values of Prandtl number $P_{r}$

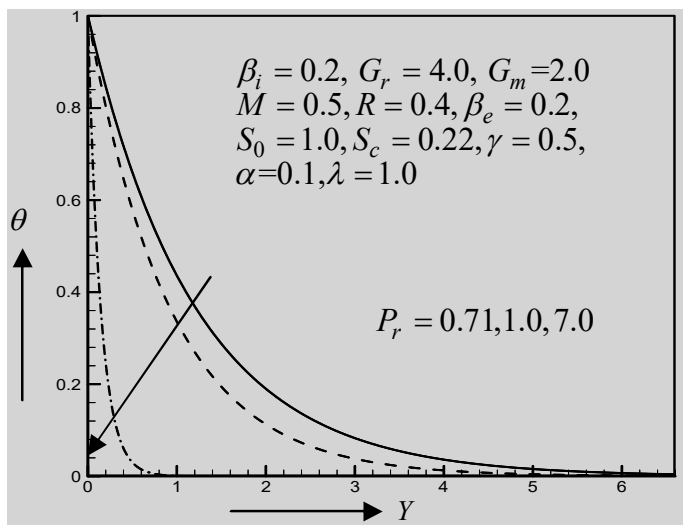

Fig. 7. Temperature profile for different values of Prandtl number $P_{r}$ 


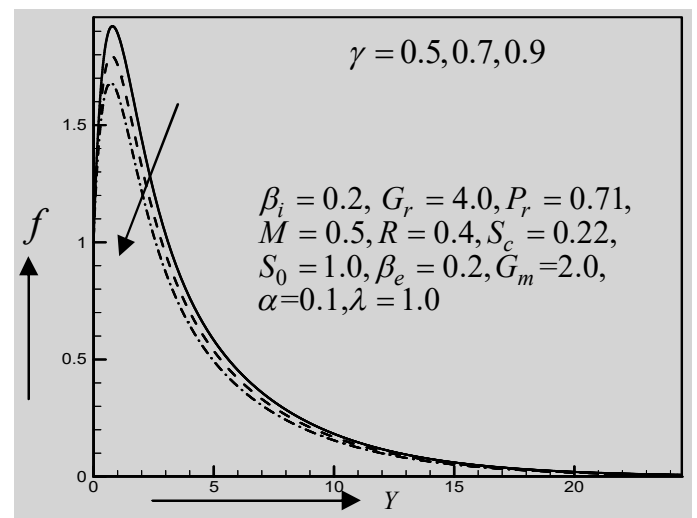

Fig. 8. Primary velocity profile for different values of permeability parameter $\gamma$

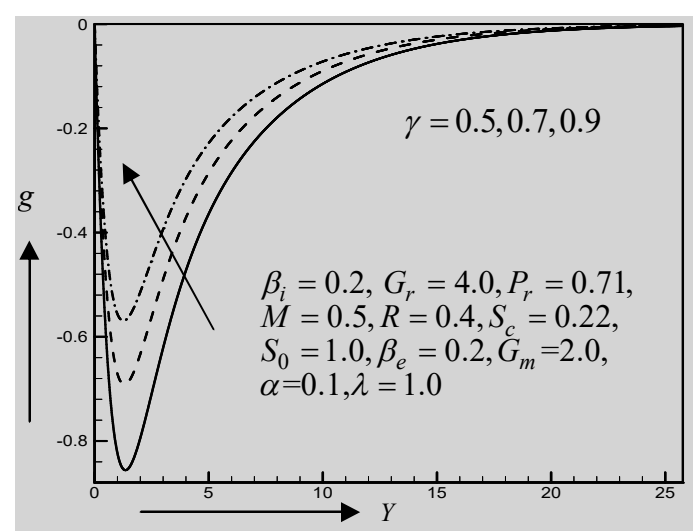

Fig. 9. Secondary velocity profile for different values of permeability $\gamma$

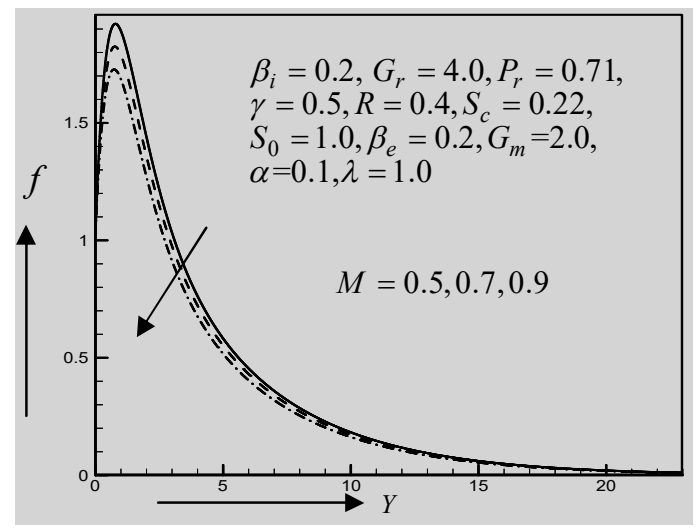

Fig. 10. Primary velocity profile for different values of magnetic parameter $M$

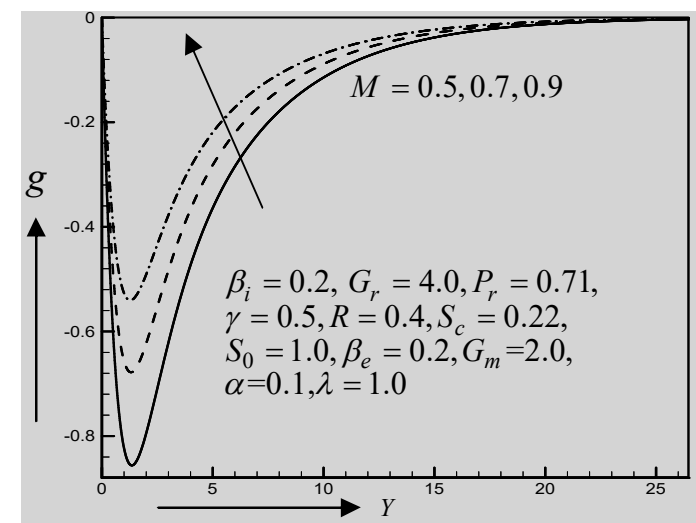

Fig. 11. Secondary velocity profile for different values of magnetic parameter $M$

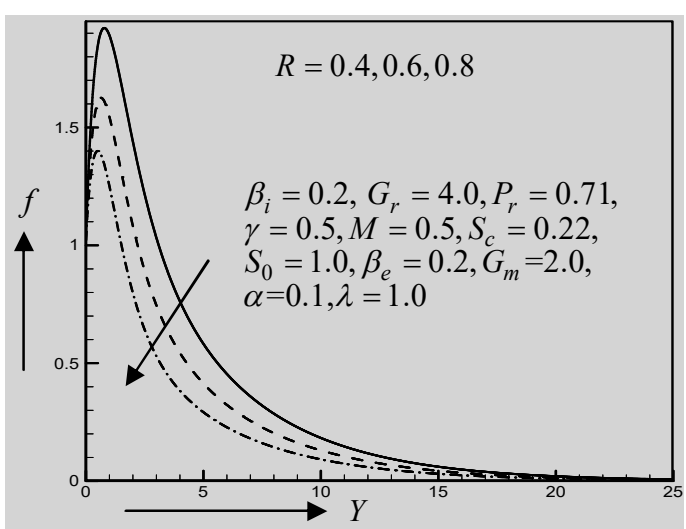

Fig. 12. Primary velocity profile for different values of rotation parameter $R$

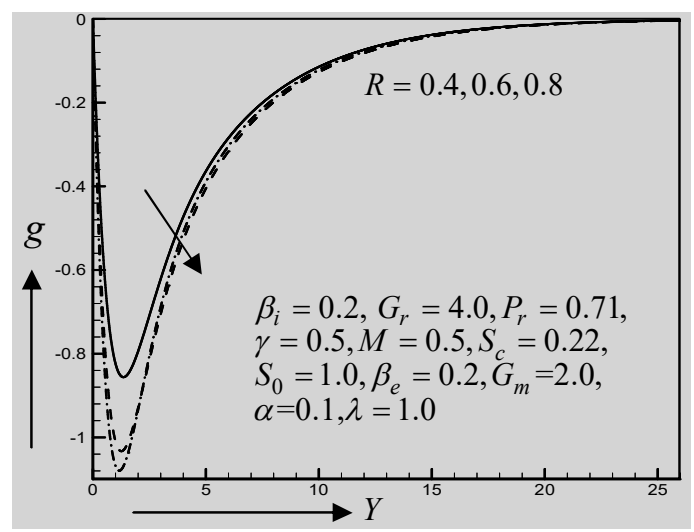

Fig. 13. Secondary velocity profile for different values of rotation parameter $R$ 


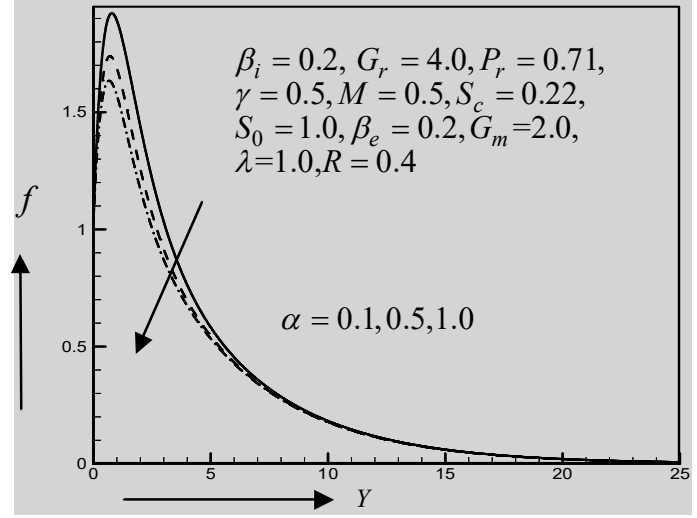

Fig. 14. Primary velocity profile for different values of heat source parameter $\alpha$

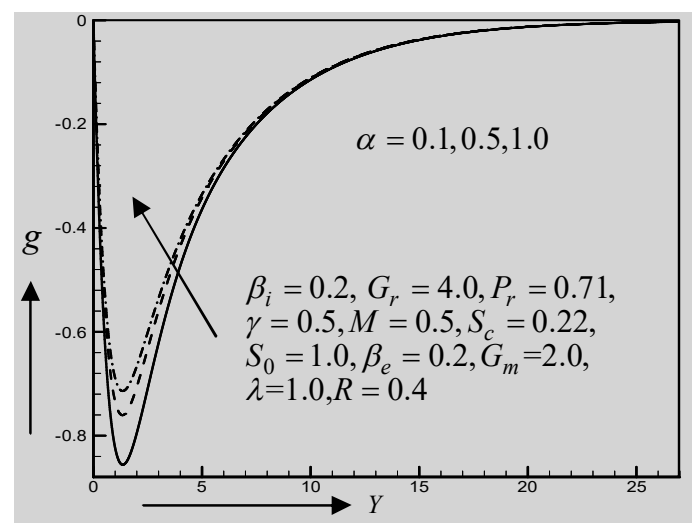

Fig. 15. Secondary velocity profile for different values of heat source parameter $\alpha$

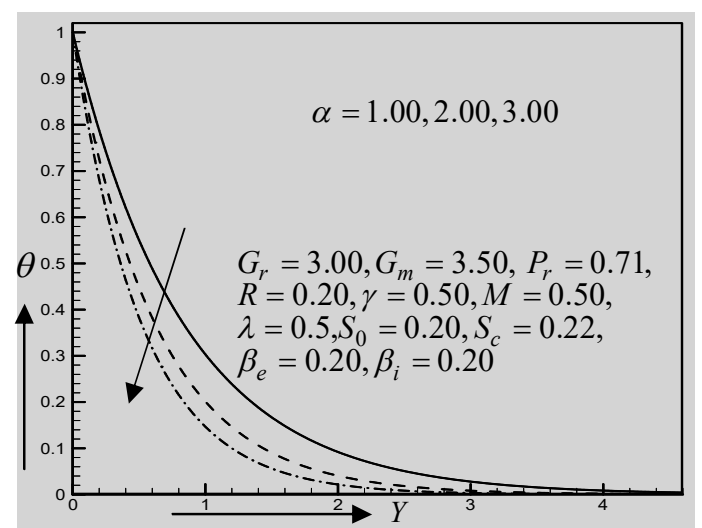

Fig. 16. Temperature profile for different values of heat source parameter $\alpha$

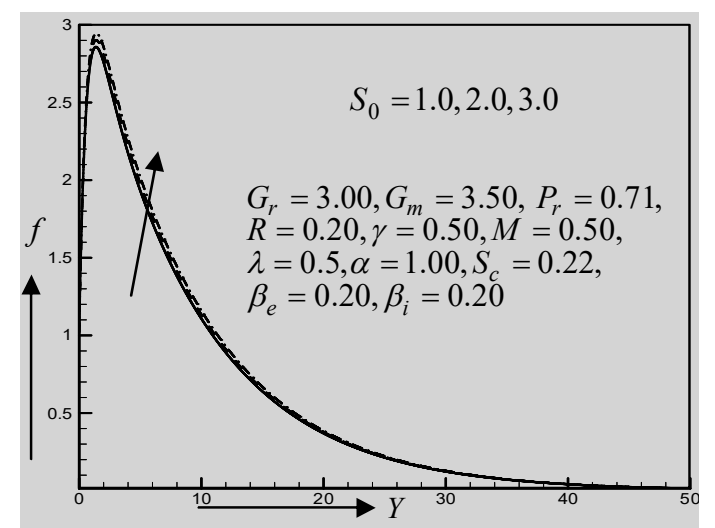

Fig. 17. Primary velocity profile for different values of Soret number $S_{0}$

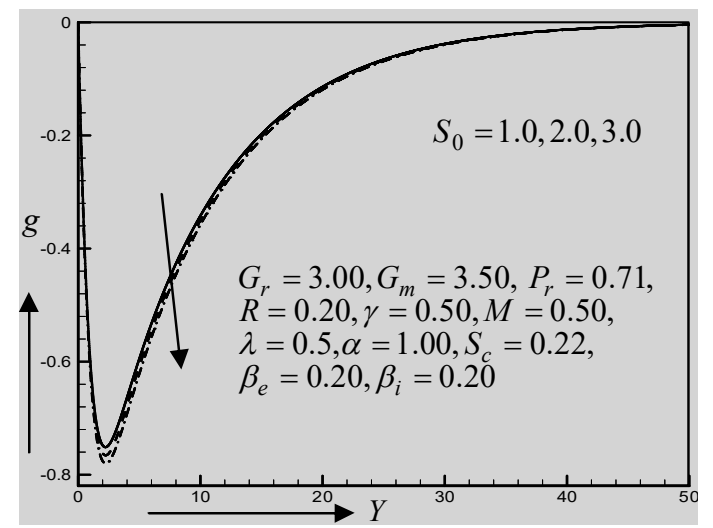

Fig. 18. Secondary velocity profile for different values of Soret number $S_{0}$

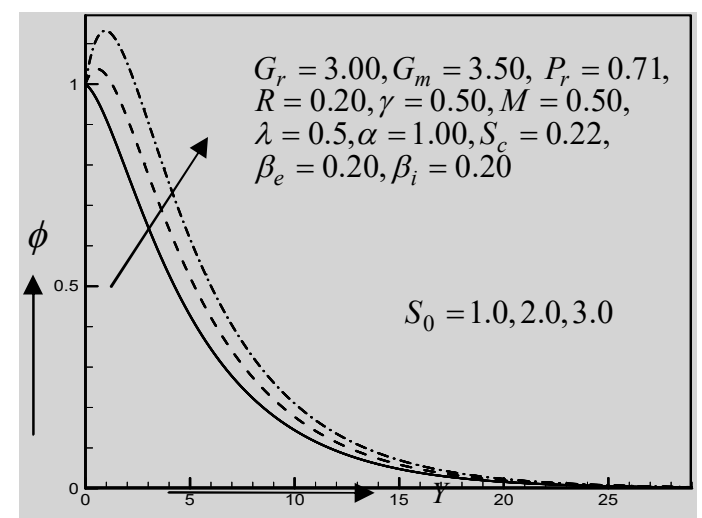

Fig. 19. Concentration profile for different values of Soret number $S_{0}$ 


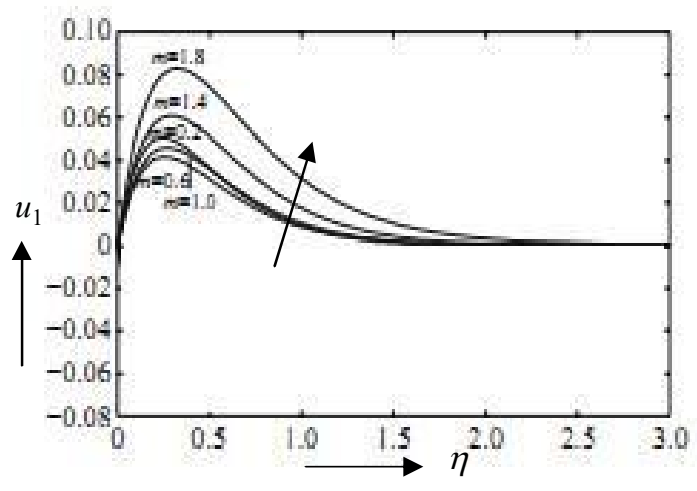

Fig. 20. Primary velocity profile of Maji et al. ${ }^{11}$ for different values of Hall parameter $m$

The present results have been compared with the results of Maji et al. ${ }^{11}$. From these two figures (Fig.20 and Fig.2) it is observed that the qualitative behavior is identically same and quantitatively different.

\section{Conclusions}

Unsteady MHD free convection and mass transfer flow through a vertical oscillatory porous plate in a rotating porous medium with Hall, ion-slip currents and heat source is studied. The governing equations are transformed to dimensionless form by using usual transformation. The dimensionless governing equations are solved by perturbation technique. Conclusions are drawn from the obtained results:

(i) The primary velocity profiles increase with the increase of Hall parameter and Soret number while it decrease with the increase of heat source, Prandtl number, permeability parameter, parameter, magnetic parameter and rotation parameter.

(ii) The secondary velocity profiles increase with the increase of heat source parameter, magnetic parameter, permeability parameter and Prandtl number while the reverse effects are seen with the increase of Hall parameter, Soret number, rotation parameter and ionslip parameter.

(iii) The temperature profiles decrease with the increase of Prandtl number and heat source parameter.

(iv) The concentration profiles increase with the increase of Soret number.

\section{References}

1. Lighthill, M, J., 1954. The reponse of laminar skin friction and heat transfer to fluctuations in the stream velocity, Proceeding of the Royal Society of London. 224, A, 1-23.

2. Soundalgekar, V. M., 1973. Free convection effects on the oscillatory flow past an infinite, vertical porous plate with constant suction. Proc. R. Soc. A 333, 25-26.

3. Soundalgekar, V. M., 1975. Free convection effects on the oscillatory flow of an electrically conducting, viscous fluid past an infinite vertical porous plate with constant and the transverse magnetic field. ZAMM. 55, 257.

4. Mittal, M. L and A. N. Bhat, 19977. Heat transfer by developing MHD flow with hall and ion-slip currents. Proceeding of the $4^{\text {th }}$ national conference on heat and mass transfer, Roorkee University, Roorkee, 247-253.

5. Soundgelkar, V. M., N. V. Vighnesam, and H. S. Takhar, 1978. Hall and ion-slip effects in MHD coutte flow with heat transfer. IEEE Transaction on Plasma Solids. 7, 178-182.

6. Venkatasiva Murthy, K. N., 1979. The effects of hall currents on the oscillatory MHD flow past a flat plate are investigated . Indian J. Pure Appl. Math. 10(9), 1051-1061.

7. Singh, A. K., 1983. Hall effects on an oscillatory MHD flow in Stokes problem past an infinite vertical porous plate. Astrophysics and space science, 93(1), 1-13.

8. Ram, P.C., 1991. Wall temperature oscillation on convection flow in a rotating fluid with hall and ion- slip currents. Astrophysics and Space Science. 175, 119-123

9. Abo-Eldahab, E. M. and M. A. Aziz, 2000. Hall and ion-slip effects on MHD free convective heat generating flow past a semi-infinite vertical plate flat plate. Physica Scripta. 61, 344-348.

10. Atti, H. A., 2004. Ion-slip on the flow due to a rotating disk. The Arabian Journal of Science Engineering. 29, 165-172.

11. Maji, S. L., A. K. Kanch, M. Guria, and R. N. Jana, 2009. Hall effects on hydromagnetic flow on an oscillating porous plate. Appl. Math. Mech. - Engl. Ed. 30(4), 503-512. 